\title{
Polypropylene meshes and other alloplastic implants for soft tissue and cartilage nasal reconstructive surgery - a literature review
}

\author{
Sabina Ionita, Serban Popescu, loan Lascar \\ Department of Plastic and Reconstructive Surgery, Emergency Clinical Hospital, Bucharest, Romania
}

ABSTRACT

BACKGROUND. The reconstructive surgery is a domain in continuous research for new techniques and alloplastic materials for replacement of complex defects. Different biomaterials are used in soft tissue reconstruction including polypropylene meshes covered with collagen, which have the best results in abdominal and pelvic surgery, but are not yet used in nasal surgery. MATERIAL AND METHODS. We analysed the studies from the literature regarding the different alloplastic implants used in nasal reconstruction surgery, their benefits and contraindications for nasal defects.

RESULTS. The most used polymers are Silicone, Medpor ${ }^{\circledR}$, Mersilene $^{\circledR}$ and polypropylene. Silicone is no longer widely used in facial reconstructive surgery because of its many complications. Medpor ${ }^{\circledR}$ (high-density polyethylene) is used for reconstruction of the facial skeleton and for aesthetic contour enhancement, including nasal reconstruction. Mersilene ${ }^{\circledR}$ (polyethylene terephthalate) is used for dorsum nasal defect reconstruction. Gore-Tex ${ }^{\circledR}$ is used for soft-tissue augmentation in the nose and is not recommended as a structural graft. Polypropylene meshes (Marlex ${ }^{\circledR}$ ) are widely used in abdominal and chest wall reconstructive surgeries, with few studies on using them in nasal cartilage reconstruction.

CONCLUSION. Nasal reconstructive surgery is a difficult part of plastic surgery than can cause many problems to the surgeon, the need for soft tissue and cartilage reconstruction are difficult to solve in a repeatedly operated nose with few autogenous graft options and complex reconstructive surgeries, especially in posttraumatic defects and revision rhinoplasties. There are many alloplastic implants that can be used with excellent results.

This paper is supported by the Sectoral Operational Programme Human Resources Development (SOP HRD), financed from the European Social Fund and by the Romanian Government under the contract number POSDRU/159/1.5/137390

KEYWORDS: polypropylene mesh, collagen coverage, biocompatibility, reconstructive surgery, alloplastic implants

\section{INTRODUCTION}

The reconstructive surgery is a domain in continuous research for new techniques and alloplastic materials for replacement of complex defects. Nasal reconstructive surgery includes many options: skin, bone and cartilage grafts, local flaps, regional flaps and free flaps and composite grafts; but, regardless of the fact that autogenous grafts are the most used materials for repairing nasal defects, many complications of these grafts occur. Different biomaterials are used in soft tissue reconstruction including polypropylene meshes covered with collagen, which have the best results in abdominal and pelvic surgery, but are not yet used in nasal surgery.

The nasal reconstructive surgery must follow the nasal aesthetic subunit principle and the reconstruction is made in three anatomical layers: the internal lining, the structural support and the skin coverage. It is important to maintain the function of the nose and the aesthetic aspect. Since it is localized in the middle of the face, the reconstruction surgery will have an important psychological role and it is important in the social relationships of the patient. It is im- 
portant to choose the best implant for the nasal reconstruction surgery because it is of great functional importance and aesthetic role for the facial symmetry.

\section{THE IDEAL BIOMATERIAL FOR NASAL RECONSTRUCTIVE SURGERY}

The ideal biomaterial which can be used in nasal reconstructive surgery must have a range of macroscopic and microscopic characteristics ${ }^{1}$.

The macroscopic characteristics of an ideal biomaterial are: compatible physical properties, retains constant shape and volume, non-resorbable ${ }^{1}$, non-allergenic $^{2}$, easily removable, retains stable position ${ }^{1}$, can be shaped to the desired form, sterilizable ${ }^{2}$, does not migrate, exchangeable, modifiable, easily available and cost-effective ${ }^{1}$. Polymers are important in soft- and hard-tissue contour restoration.

The microscopic characteristics are: no or minimal inflammatory response or surface contamination, infection resistant, non-degradable, no disease transmission and non-carcinogenic ${ }^{1}$. The ideal alloplast must be chemically inert, biocompatible ${ }^{3}$, not physically modified by soft tissue; it should elicit no chronic inflammatory reaction or foreign body reaction, integrate into soft tissue or bone, resist mechanical strains. The site of implantation must meet the following conditions: no infection, no poor soft-tissue coverage, no poor blood supply from scarring, no history of radiotherapy, no soft-tissue tension ${ }^{2}$.

The available synthetic meshes are considered noncarcinogenic, but the chronic inflammation that could be determined by the meshes can cause cancer. There are cited several cases of squamous-cell carcinoma (SCC) of the abdominal wall in patients with longterm mesh infection ${ }^{4}$.

The ideal biomaterial should mimic the colour, consistency and aesthetic aspect of the recipient area, permitting tissue ingrowth ${ }^{5}$.

The cartilage is an immunologically privileged tissue because of its matrix through which antibodies and lymphocytes are unable to pass; but, if the matrix is damaged, the cartilage is susceptible to immunogenic rejection. So the crushed allografts are more quickly resorbed than block allografts. This explains the slow but constant resorption of the cartilage allografts (homologous cartilage) ${ }^{2}$. The cartilage graft rejection is primarily a cell-mediated reaction with minor contribution of beta-lymphocytes ${ }^{6}$. Alloplastic material has no donor site scars or morbidity, a limitless source, no operative time for harvesting of the graft and no potential for disease transmission, with low operative time and major patient satisfaction ${ }^{2}$.

In nasal reconstructive surgery, the ideal biomaterial for cartilage repair is the autogenous cartilage graft, but the supply of autogenous cartilage is, in many situations, limited and there is an important morbidity at the donor site ${ }^{7}$.

Many synthetic materials are used in reconstructive surgery and also in nasal reconstructive surgery, including Medpor ${ }^{\circledR}$ (porous polyethylene), Gore-Tex ${ }^{\circledR}$, polypropylene and many others. But, there are many complications that can occur when using these materials $^{7}$.

Implant characteristics for using them as nasal implants are pore size, consistency and malleability. Meshed implants have host tissue ingrowth which minimizes infection. Porous implants have a smaller proportion of empty space in comparison to meshed implants. They allow host tissue ingrowth that provide stability, but the ingrowth is lower than of the meshed implants; so, the implant removal, if needed, is less complicated ${ }^{8}$. There are many options regarding the alloplastic materials in nasal surgery, but it must be reinforced that the ideal nasal implant is an autogenous implant and the allogenic polymers must be used with great care ${ }^{8}$.

A large variety of alloplastic materials have been used for nasal dorsal reconstruction. The thin dorsal soft tissue and proximity to the nasal cavities pose challenges to the use of alloplasts ${ }^{3}$, limiting their use to mainly the dorsum nasi ${ }^{9}$.

The most used polymers in nasal augmentation are Gore-Tex ${ }^{\circledR}$, polyamide mesh and Silicone ${ }^{10}$.

Silicone (polydimethylsiloxane), polyurethane $(\mathrm{PU})$, polyethylene (PE), polyethylene terephthalate (PET), polytetrafluoroethylene (PTFE), methylmethacrylate (MMA) and other thermoplastic polymers have a high degree of biocompatibility and resistance to degradation, except under extreme heat, UV radiation or hydrolysis ${ }^{11}$. Hydrophilic polymers are more susceptible to degradation than hydrophobic polymers ${ }^{2}$.

\section{Silicone (polydimethylsiloxane)}

Silicone is the most commonly used implant for nasal augmentation; it is inert and determines little tissue reaction, it develops around itself a fibrous capsule that stabilizes the implant and it can be colonized with bacteria. In many countries, this implant is not used at present because of the many complications it leads to: exposure, migration, calcification, extrusion, inflammation ${ }^{12}$.

Silicone takes the form of fluid, gel or rubber ${ }^{13}$. As fluid or gel, it determines a cellular response as a foreign-body reaction (silicone inclusions in giant cells surrounded by neutrophils, plasma cells, and lymphocytes $)^{14}$. So, it should never be injected for soft-tissue augmentation. This foreign-body reaction also forms a capsule around the implant. Silicone rubber implant sheets are used for malar, chin and nasal augmenta- 
tion. Silicone elastomers are stable to heat and oxygen, sterilizable with ethylene oxide gas, irradiation or steam $^{15,16}$, non-porous (they resist tissue ingrowth), chemically inert, non-biodegradable, nontoxic and non-allergenic, good electrical insulators, versatile by taking many shapes and malleable intraoperatively. Silicone elastomers are also used for making the shell of tissue expanders².

\section{Medpor $^{\circledast}$ (high-density polyethylene)}

High-density molecular weight polyethylene (HDPE) is biocompatible, nonresorbable, with a high tensile strength and can cause less underlying bone resorption than other implants ${ }^{17}$. It allows both bony and soft-tissue ingrowth. It has low infection rates. HDPE cannot be used in sites that have low vascularization, in sites where soft-tissue coverage is poor and in the presence of infection. It has been used in chin, nasal, malar and mandibular angle augmentation, and for trauma surgery ${ }^{2}$.

Medpor $^{\circledast}$ (high-density polyethylene), introduced in the 1970 s, elicits minimal foreign body reaction ${ }^{18}$. Its interconnecting pore structure allows for fibrovascular ingrowth and integration of the surrounding tissue. It was widely used in rhinoplasty and revision rhinoplasty, but nowadays it is rarely used because of its important complications. Medpor ${ }^{\circledast}$ can be used as columellar strut, dorsal, spreader, rim and batten grafts and in patients with septal perforation. The complications cited are lateral movement of implant, fistula formation, skin thickness, erythema, abscess formation, extrusion of the implant (inserting it close to the incision site is a risk factor for extrusion) and infection ${ }^{19}$.

When the mesh was used for septal perforation no extrusion or other complications were described. It does not lead to the problems associated with solid and nonporous grafts. Medpor ${ }^{\circledR}$ implants determine excellent contouring, increased mechanical stability, decreased risk of implant migration and infection. They improve nose function and can resist to trauma and scar contracture force. These types of implants are better than cartilage grafts, because they do not have cartilage memory problem and do not lead to recurrence. The most frequent complications of this mesh occur in rim grafts, so it is not indicated to use Medpor $^{\circledR}$ implants. Another contraindication of this implant is the strut grafts. It can be used as dorsal and spreader grafts and for small-size septal perforations ${ }^{19}$.

Medpor $^{\circledR}$ determines rapid ingrowth of vascularized tissue with collagen deposition, leading to implant stabilization. It can be sculptured into the desired shape, but it can create an unnatural appearance over the dorsum nasi because of its stiffness. They prove to have good results in dorsum nasi augmentation due to their long-term structural stability and lack of resorption ${ }^{3}$.
Medpor $^{\circledR}$ implant used as a skeleton substitute is biocompatible and porous. For this reason, it has been used for facial skeleton reconstruction and for aesthetic contour enhancement. The use of alloplast implants for nasal reconstruction is controversial in the literature because their immune tolerance is not well known. Medpor ${ }^{\circledR}$ has been used for chin and malar augmentation. The histopathological results revealed soft tissue ingrowth and collagen deposition with formation of vascularization. The complications were few, including extrusion and infections, but $91 \%$ of the implants remained unchanged ${ }^{20}$.

Considering the results and the data reported in the literature, Medpor ${ }^{\circledR}$ seems to be the best alloplastic implant for facial bone substitute with high patient satisfaction ${ }^{20}$.

\section{Mersilene $^{\circledast}$ (high density polyethylene)}

Mersilene $^{\circledast}$ (high density polyethylene) is used in the augmentation of the temporal fossa. This mesh can be folded to recreate the anatomical configuration, contours naturally to the defect, it is cost-effective with very good aesthetic results and low complication rate, including extrusion, exposure of the implant and infection. This type of implant has proved to be better than HDPE implants for the augmentation of the temporal fossa ${ }^{21}$. Mersilene ${ }^{\circledast}$ provides a natural appearance, but with a high infection rate and an extensive fibroblast ingrowth. It is also used for the augmentation of the dorsum nasi ${ }^{22}$. Polyester mesh (Mersilene ${ }^{\circledR}$ ) is preferred as nasal implant in some studies, even if it has a $3.5 \%$ infection rate ${ }^{8}$.

Porous high-density polyethylene (PHDPE) is widely used as a nasal implant, for chin and malar augmentation and for orbital reconstruction. It has been widely used in facial reconstructive surgery for softtissue augmentation and structural support, but it is rigid and it has documented infection rate ${ }^{8}$.

Mersilene $^{\circledast}$ is a stable and easily shaped mesh for dorsum nasal defect reconstruction, with contradictory results depending on the study. Extensive fibroblast ingrowth stabilizes the mesh, but it has been less preferred for dorsum nasi reconstruction than Gore$\mathrm{Tex}^{\circledR 23}$.

Polyethylene terephthalate is used in reconstructive surgery including arterial prostheses. PET is used for abdominal and chest wall reconstruction and for genioplasty ${ }^{24}$, head and neck surgery and facial and nasal augmentation $^{25}$.

\section{Gore-Tex ${ }^{\circledR}$ (expanded polytetrafluoroethylene)}

PTFE (polytetrafluoroethylene) was introduced to plastic surgery as Proplast ${ }^{\circledR}$ facial implants. It forms a fibrous tissue layer on the bone ${ }^{26}$, but can cause foreign body reaction. For subcutaneous augmentation, PTFE is available as Gore-Tex ${ }^{\circledR}$, which gives good 
strength, is biologically inert, non-allergenic, non-carcinogenic, its pores allow for tissue ingrowth and has low infection rates ${ }^{2}$. It has been used clinically in facial aesthetic surgery for $\mathrm{lip}^{27}$, $\operatorname{chin}^{28}$, nasal ${ }^{29-31}$, malar $^{28}$ and forehead augmentation ${ }^{2}$.

If extensive augmentation of the dorsum nasi is needed, the autogenous grafts cannot be used, so the need for an alloplastic graft is great. Gore-Tex ${ }^{\circledR}$ is a promising material. Gore-Tex ${ }^{\circledast}$ is used extensively in vascular surgery and has a high biocompatibility. It has been used for the first time as a vascular prosthesis in $1972^{32}$. This polymer was used in many revision rhinoplasties where there were few options for autogenous grafts. In comparison with Mersilene ${ }^{\circledast}$, it has poor chronic inflammation and foreign-body reaction ${ }^{33}$. This polymer can be used extensively in facial reconstructive surgery because it fills up the prominent skin creases and bony depressions. For this reason, it is widely used for chin augmentation. There have been few complications after using Gore-Tex ${ }^{\circledR 34}$, the surgical approach and technique being very important for this aspect. It has low migration rate. Gore-Tex ${ }^{\circledR}$ is used with excellent result in general and vascular surgery. For the rhinoplasty surgery future studies are needed to prove the long-term success and morbidity ${ }^{22}$.

Gore-Tex $^{\circledast}$ is used for soft-tissue augmentation in the nose and it is not recommended as a structural graft. It has an overall complication rate of $3.2 \%$. It has sufficient ingrowth to stabilize the implant, but it allows the graft removal if it has encountered complications. A new implant of Gore-Tex ${ }^{\circledR}$ reinforced with fluorinated ethylene propylene enhances pliability and firmness. It has favourable vascular migration and limited capsule formation in an experimental study ${ }^{8}$.

Gore-Tex ${ }^{\circledast}$ is an excellent implant for chin augmentation, especially using the dual plane Gore-Tex ${ }^{\circledR}$ chin augmentation procedure ${ }^{35}$.

Gore-Tex ${ }^{\circledast}$ is used in general and vascular surgery. It has low tissue reactivity, excellent biocompatibility and reasonable cost. Its microporous composition encourages tissue ingrowth, which confers stability to the implant. Due to these characteristics, the implant firmly adheres to the surrounding tissues and prevents migration. It can fill or contour a defect, but it provides little structure at the implant site ${ }^{3}$. In a multicenter, retrospective study on 309 patients who underwent dorsal nasal augmentation with Gore-Tex implants, $3.2 \%$ of the implants were infected in the patients with nasal septal perforation ${ }^{36}$.

\footnotetext{
Polypropylene meshes and polypropylene covered with collagen meshes

Polypropylene meshes are widely used in abdominal and chest wall reconstructive surgeries, with few studies on using them in nasal cartilage reconstruction.
}

Yucebas K. et al evaluated the effectiveness and biocompatibility of the polypropylene mesh in experimental rabbits for nasal septal perforations. The surgical management includes the advancement of the mucosal flaps with cartilage interpositional grafts between the mucosal walls. Polypropylene meshes are very frequently used for inguinal hernia repair because it is biocompatible and stable. The polypropylene meshes were placed between the two mucosal flaps. The polypropylene mesh is biocompatible, cheap and manipulable in terms of dimensions. The mucosa could proliferate on this mesh, and it could cover the edges of the defect as well as the cartilage, with no rejection in the laboratory rabbits. The mesh caused limited fibrosis and foreign body granuloma stimulated by the chronic inflammatory response, with no rejection or infection. The biocompatibility of this mesh with the septal mucosa was very high ${ }^{37}$.

Pierce L.M. et al compared the use of polypropylene meshes covered with collagen and simple polypropylene meshes in abdominal wall reconstructive surgery, regarding the expression of genes responsible for formation cells, which have a role in wound healing. They studied the genes in laboratory rats, created the abdominal wall defects and repaired them with polypropylene meshes covered with collagen and simple polypropylene meshes. The histological, immunohistochemical and mRNA analyses show that, one week after implantation, composite meshes elicited a greater inflammatory response than simple polypropylene meshes and an increased mRNA expression of 4 proinflammatory cytokines. But after 90 days the inflammatory response and the expression of cytokines were comparable in both meshes, showing similar results for both types. Both meshes can be used in abdominal wall reconstruction with great results ${ }^{38}$.

Goulart F. et al performed an immunohistochemical analysis of the polypropylene mesh coated with highly purified collagen gel in laboratory rats. Those meshes caused important changes in angiogenesis and in immune reaction of metalloproteinase around mesh implants. There was a higher vessel density compared to simple polypropylene meshes, more stability of MMP-3 with the decrease of MMP-3 after 14 and 90 days and a decrease in average density of MMP-3 after 21 days in the simple polypropylene meshes and after 90 days in the polypropylene meshes covered with collagen. This can improve the mesh biocompatibility for pelvic floor surgery ${ }^{39}$.

Collagen has a cell ingrowth enhancement potential and, because it is a natural polymer, it has a unique biocompatibility. There are studies that explain that using a collagen/polypropylene mesh intraperitoneally can decrease visceral adhesion. An acetic acid extracted collagen coating experiment was developed in experimental rats and the results were very good, 
with few complications and a good biocompatibility of the polypropylene mesh ${ }^{40}$.

Collagen/ polypropylene composite meshes (Parieten ${ }^{\circledR}$ ) can increase the infection rate in experimental rats, but it has very good biocompatibility and low adhesion rate in laparoscopic incisional hernia repair ${ }^{41}$.

In an observational study on patients with a stage 2 cystocele with or without associated apical or posterior vaginal wall collapse, collagen/polypropylene composite meshes were used, with a high recurrence rate and a high exposure rate, so the results were poor. The composite meshes will not be used for this type of defects ${ }^{42}$.

An experimental study on laboratory rabbits compared the use of polyester with absorbable layer prosthesis and collagen-elastin/polypropylene prosthesis for the repair of abdominal wall defects. The results were comparable, with approximately the same adhesion rate formation. The polyester mesh with absorbable layer has a higher deposition of collagen type I and collagen type III. The presence of metalloproteinases was approximately the same, expression of MMP1, MMP-8 and MMP-13 were studied, with low difference in MMP-13 at 60 days. The expression of MMP-13 increased in the polyester mesh with absorbable layer prosthesis group ${ }^{43}$.

The polypropylene mesh provides mechanical strength, a predictable host response and it is widely used for ventral hernia and pelvic organ prolapse. It has many complications such as chronic local tissue inflammatory response and dense fibrous tissue deposition. The biologic scaffold materials composed of extracellular matrix (ECM) are rapidly degraded invivo and are associated with constructive remodelling and minimal fibrosis. If the polypropylene mesh is coated with ECM, it lowers the inflammatory response. At 180 days, there is a decrease in the density of collagen and the amount of mature collagen deposited between the mesh fibers, in comparison with polypropylene meshes. ECM coating lowers the chronic inflammatory response and associated scar tissue deposition characteristic of polypropylene ${ }^{44}$.

There are studies in abdominal surgery that compare a new mesh, Parietex composite ventral patch, with Ventralex ST hernia patch and Proceed ventral patch in laboratory rabbits for the study of umbilical hernia repair. Adhesion formations were greater for Proceed ventral patch compared to Ventralex and Parietex. The Proceed ventral patch has tissue-integrated adhesions affecting the intestinal loops and greater macrophage response. All the implants have similar patterns of collagen I and III deposition. Parietex and Ventralex have excellent mesothelialization with minimum adhesion formation ${ }^{45}$.

The collagen-coated non-absorbable monofilament polypropylene mesh (Avaulta plus ${ }^{\circledR}$ ) for cystocele has no benefit compared with conventional anterior colporrhaphy, but elicits greater costs. The objective cure rate was $39.8 \%$ with the conventional method and $88.1 \%$ with the alloplastic method, with better anatomical outcome. It has high exposure rate compared to conventional surgery. This study proves that coated meshes do not have any advantages compared to the uncoated meshes in pelvic surgery. There was a high rate of urinary incontinence because the mesh can overcorrect the position of the bladder neck and/or urethra $^{46}$. This study is not relevant for nasal reconstruction because the complications that are cited occur because of the type of surgery, not because of the mesh used.

The collagen-covered meshes (Collamend $^{\circledR}$, Surgisis ${ }^{\circledR}$ and Permacol $^{\circledR}$ ) can be used with good results in abdominal surgery. These collagen-covered meshes, in contact with Staphylococcus aureus and Staphylococcus epidermidis, show greater bacterial loads than the polytetrafluoroethylene mesh Preclude ${ }^{\circledR}$. There is no benefit using them if there is an infection at the implantation site. Preclude ${ }^{\circledR}$ has better results in a contaminated area $^{47}$.

For pelvic floor reconstruction, it is researched the $0.5 \%$ chitosan-coated or high molecular weight polypropylene mesh (Ch-PPM). This mesh determines preferential attachment of myoblasts over fibroblasts. Ch-PPM has an early macrophage phenotype response that determines more functional outcomes ${ }^{48}$.

Fibroblast or the mesenchymal stem cell (MSC) coats Parietex $^{\circledR}$ (polyester), SoftMesh ${ }^{\circledR}$ (light weight monofilament polypropylene), TIGR ${ }^{\circledR}$ (polylactide composite) or Strattice ${ }^{\circledast}$ (porcine dermal collagen). MSC coating lowers secretion of cytokines IL-1beta, IL-6 and VEGF by macrophages, with the exception of SoftMesh ${ }^{\circledR}$. Intimate interactions between cell-coated meshes and macrophages were cited. Cytokine response to fibroblast coating varies, but MSC coating lowers the immunogenic effect of both synthetic and biologic meshes. Cell coating appears to affect mesh biocompatibility and may become a key process in mesh evolution ${ }^{49}$.

In other studies, SoftMesh ${ }^{\circledast}$, Parietex-TET ${ }^{\circledast}$, TIGR $^{\circledast}$, Marlex $^{\circledR}$ (heavy weight monofilament polypropylene) and Strattice ${ }^{\circledast}$ were coated with human dermal fibroblasts (HFs), rat kidney polypropylene (NRKs) and rat MSCs. These covered meshes modulate the host response to various synthetic and biologic meshes ${ }^{50}$.

In pelvic reconstructive surgery for pelvic organ collapse, collagen-coated polypropylene mesh has been used, with little degeneration of the collagen barrier and with good clinical results ${ }^{51}$.

Polypropylene $\left(\right.$ Marlex $\left.^{\circledR}\right)$ is widely used in large scale for chest and abdominal wall reconstruction. For this polymer, explantation is very difficult because of the tissue ingrowth that occurs. It is highly porous, like Medpor $^{\circledR}$, and therefore has the risk of infection ${ }^{2}$. 


\section{DISCUSSIONS}

The aim of nasal reconstructive surgery is to repair the defect with preservation of the nasal function and its aesthetic aspect. The surgeon must apply the nasal aesthetic subunits principle and he/she has to repair the three layers of the nose: internal lining, structural support and skin covering. Autogenous cartilage is the best graft for structural reconstruction. But for revision nasal reconstruction where the autogenous cartilage is in short supply, the alloplastic implant has been used with good results ${ }^{52}$.

Gore-Tex ${ }^{\circledast}$ is used for the augmentation of the dorsum nasi or lateral walls, premaxilla graft, tip or supratip graft. According to Ham in a study performed in 2003 , the rate of infection was of $0-10 \%{ }^{52}$. Gore$\mathrm{Tex}^{\circledR}$ is widely used in rhinoplasty surgery, but recent histological studies demonstrated that they determine greater foreign-body reaction than suspected ${ }^{53}$.

Silicone is used for dorsum nasi augmentation and as a columellar strut. Ahn, in 2004, used this implant with excellent results, with few complications including misalignment of the silicone dorsal implant and recurrent dorsal edema ${ }^{52}$.

Porous high-density polyethylene $\left(\right.$ Medpor $\left.^{\circledR}\right)$ can provide much structural support. It has low rates of exposure and infection. Pre-formed implants exist (dorsal implant, columellar strut and external valve battens) and can be molded as desired ${ }^{52}$. Its use remains controversial because of its important complications: extrusion and infection ${ }^{53}$. It can be used for structural support. It is used for trauma surgery, not only in rhinoplasties ${ }^{54}$.

Mersilene $^{\circledR}$ (polyethylene terephthalate) is used for volume correction in nasal reconstruction, but it cannot be used for structural support. Connective tissue that grows around it attaches the mesh ${ }^{54}$. It is an excellent alloplastic implant for facial skeleton augmentation if autogenous grafts cannot be harvested. It can be rigid and sometimes is palpated extraorally and infection is a complication cited ${ }^{55}$.

Tissue engineered cartilage is the technology of the future. Chondrocytes are harvested from the nasal septum and cultivated for implanting on a biodegradable scaffold and then implanting them in the nasal dorsum $^{52}$.

Polypropylene is rarely used in nasal reconstructive surgery with few studies on its advantages and disadvantages as a nasal implant. Polypropylene meshes are widely used in abdominal wall reconstruction and pelvic prolapse surgery ${ }^{56}$. Collagen coated polypropylene meshes are used in the same field, but future studies will determine if they can be used with excellent results in nasal reconstructive surgery.

\section{CONCLUSIONS}

There are many studies that confirm the supremacy of using polypropylene meshes covered with collagen in comparison with polypropylene meshes in abdominal and chest wall reconstruction. Despite the widely use of alloplastic implants in nasal surgery, polypropylene meshes are rarely used for nasal reconstruction, Gore-Tex ${ }^{\circledast}$ is used at a larger scale than other biomaterials. Some authors cite that polypropylene meshes can be used for nasal septal reconstruction with good results.

Coverage of polypropylene meshes with collagen gives them a better biocompatibility, with fewer complications and better integration of the implants. Collagen is a natural polymer, which is part of the original cartilage that we want to reconstruct; it is biocompatible and does not enhance foreign-body reaction. The coverage of the mesh with this polymer can only give better results in nasal reconstructive surgery.

Nasal reconstructive surgery is a difficult part of plastic surgery than can cause many problems to the surgeon; the need for soft tissue and cartilage reconstruction are difficult to solve in a multiple operated nose with few autogenous graft options and complex reconstructive surgeries, especially in posttraumatic defects and revision rhinoplasties. Many alloplastic implants are used in nasal reconstructive surgeries, but there is not any ideal biomaterial which can be used in these situations where the recipient bed is vicious, it has many scars from previous surgeries and low vascularization. The alloplastic implants in this condition have also poor results, but future studies in nasal reconstructive surgeries regarding polypropylene meshes covered with collagen could have excellent results.

Acknowledgement: This paper is supported by the Sectoral Operational Programme Human Resources Development (SOP HRD), financed from the European Social Fund and by the Romanian Government under the contract number POSDRU/159/1.5/137390

\section{REFERENCES}

1. Vuyk H.D., Adamson P.A. - Biomaterials in Rhinoplasty. Clin. Otolaryngol., 1998;23:209-217.

2. Lipschitz A.H., Kenkel J.M. - Implantation bone, cartilage, and alloplasts. In: Barton F.E. (ed.) - Selected readings in plastic surgery. Vol. 10(2), Dallas: Selected Readings in Plastic Surgery, 2004.

3. Dresner H.S., Hilger P.A. - An Overview of Nasal Dorsal Augmentation. Semin Plast Surg., 2008 May;22(2):65-73.doi:10.1055/s-2008-1063566.

4. Birolini C., Minossi J.G., Lima C.F., Utiyama E.M., Rasslan S. - Mesh cancer: long-term mesh infection leading to squamous-cell carcinoma of the abdominal wall. Hernia., 2014 Dec;18(6):897-901.

5. Graham B.S., Thiringer J.K., Barrett T.L. - Nasal tip ulceration from 
infection and extrusion of a nasal alloplastic implant. J Am Acad Dermatol., 2001 Feb;44(2 Suppl):362-4.

6. Welsh K.I., Burgos H., Batchelor J.R. - The immune response to allogeneic rat platelets; Ag-B antigens in matrix form lacking Ia. Eur J Immunol., 1977;7(5):267-72.

7. Romo T. 3rd, Sclafani A.P., Sabini P. - Use of porous high-density polyethylene in revision rhinoplasty and in the platyrrhine nose. Aesthetic Plast Surg., 1998 May-Jun;22(3):211-221.

8. Romo T. 3rd, Pearson J.M. - Nasal implants. Facial Plast Surg Clin N Am., 2008 Feb;16(1):123-132.

9. Lovice D.B., Mingrone M.D., Toriumi D.M. - Grafts and implants in rhinoplasty and nasal reconstruction. Otolaryngol Clin North Am., $1999 \mathrm{Feb} ; 32(1): 113-41$.

10. Ousterhout D.K., Stelnicki E.J. - Plastic surgery's plastics. Clin Plast Surg., 1996;23(1):183.

11. Kuttenberger J.J., Hardt N. - Long-term results following reconstruction of craniofacial defects with titanium micro-mesh systems. J Craniomaxillofac Surg., 2001 Apr;29(2):75-81.

12. Yanaga H., Koga M., Imai K., Yanaga K. - Clinical application of biotechnically cultured autologous chondrocytes as novel graft material for nasal augmentation. Aesthetic Plast Surg., 2004 Jul-Aug;28(4):212-21.

13. Gear A.J., Lokeh A., Aldridge J.H., et al. - Safety of titanium mesh for orbital reconstruction. Ann Plast Surg., 2002; 48:1-7; discussion 7-9.

14. Brown B.L., Neel H.B. 3rd, Kern E.B. - Implants of Supramid, Proplast, Plasti-Pore, and Silastic. Arch Otolaryngol., 1979 Oct;105(10):605-9.

15. Holmes R.E. - Alloplastic implants. In: McCarthy J.G. (ed) - Plastic Surgery. Philadelphia, WB Saunders, 1990;p.698-703.

16. Deva A., Merten S., Chang L. - Silicone in nasal augmentation rhinoplasty: a decade of clinical experience. Plast Reconstr Surg., 1998;102(4):1230-1237.

17. Hunt J., Salomon J. - Augmentation mammoplasty. Selected Read Plast Surg., 2002;9(28):1-35.

18. Mohammadi S.H., Ghourchian S.H., Izadi F., Daneshi A., Ahmadi A. Porous high-density polyethylene in facial reconstruction and revision rhinoplasty: a prospective cohort study. Head Face Med., 2012;8:17.

19. Mohammadi S., Mohseni M., Eslami M., Arabzadeh H., Eslami M. - Use of porous high-density polyethylene grafts in open rhinoplasty: no infectious complication seen in spreader and dorsal grafts. Head Face Med., 2014;10:52.doi:10.1186/1746-160X-10-52.

20. Niechajev I. - Facial Reconstruction Using Porous High-Density Polyethylene (Medpor): Long-Term Results. Aesthetic Plastic Surgery, 2012August;36(4):917-927.

21. Atherton D.D., Joshi N., Kirkpatrick N. - Augmentation of temporal fossa hollowing with Mersilene mesh. Journal of Plastic, Reconstructive \& Aesthetic Surgery, 2009;63(10):1629-34.doi: 10.1016/j. bjps.2009.09.022

22. Lohuis P.J.F.M., Watts S.J., Vuyk H.D. - Augmentation of the nasal dorsum using Gore-Tex®: intermediate results of a retrospective analysis of experience in 66 patients. Clin. Otolaryngol., 2011;26:214-217.doi: 10.1046/j.1365-2273.2001.00453.x

23. Adamson P.A. - Grafts in rhinoplasty: autogenous grafts are superior to alloplastic. Arch Otolaryngol Head Neck Surg., 2000 Apr;126(4):561-2.

24. Nguyen P.N., Sullivan P. - Advances in the management of orbital fractures. Clin Plast Surg., 1992 Jan;19(1):87-98.

25. Ousterhout D.K. - Prosthetic Forehead Augmentation. In: Ousterhout DK (ed) - Aesthetic Contouring of the Craniofacial Skeleton. Boston, Little Brown, 1991;p.199- 219.

26. Brand K.G., Johnson K.H., Buoen L.C. - Foreign body tumorigenesis. CRC Crit Rev Toxicol., 1976;4(4):353-394.

27. Staffel G., Shockley W. - Nasal implants. Otolaryngol Clin North Am., 1995;28:295-308.
28. McCollough E.G., Hom D.B., Weigel M.T., Anderson J.R. Augmentation mentoplasty using Mersilene mesh. Arch Otolaryngol Head Neck Surg., 1990;116(10):1154-1158.

29. Mole B. - The use of Gore-Tex implants in aesthetic surgery of the face. Plast Reconstr Surg., 1992;90(2):200-206.

30. Linder R.M. - Permanent lip augmentation employing polytetrafluoroethylene grafts. Plast Reconstr Surg., 1992;90 (6):1083-1090; discussion: 1091-1092.

31. Stoll W. - The use of polytetrafluoroethylene for particular augmentation of the nasal dorsum. Aesthetic Plast Surg., 1991;15(3):233-236.

32. Soyer T., Lempinen M., Cooper P., Norton L., Eiseman B. - A new venous prosthesis. Surgery, 1972;72(6):864-872.

33. Maas C.S., Gnepp D.R., Bumpous J. - Expanded polytetrafluoroethylene (Gore-Tex soft-tissue patch) in facial augmentation. Arch. Otolaryngol. Head Neck Surg., 1993;119(9):1008-1014.

34. Godin M.S., Waedman S.R., Johnson C.M. Jr. - Nasal augmentation using Gore-Tex. A 10-year experience. Arch. Facial Plast. Surg., 1999;1(2):118-121.

35. Kim B.J., Lim J.W., Park J.H., Lee Y.H. - Dual plane augmentation geniopasty using Gore-Tex chin implants. Arch Craniofac Surg., 2014 Aug; $15(2): 82-88$.

36. Owsley T.G., Taylor C.O. - The use of Gore-Tex for nasal augmentation: a retrospective analysis of 106 patients. Plast Reconstr Surg. 1994;94(2):241-248.

37. Yücebaș K., Taskin U., Oktay M.F., at al. - The Results of Polypropylene Mesh Usage for Nasal Septal Perforation Repair: An Experimental Study. Available at: http://www.researchposters.com/Posters/ AAOHNSF/AAO2014/SP493.pdf

38. Pierce L.M., Asarias J.R., Nguyen P.T., Mings J.R., Gehrich A.P. Inflammatory cytokine and matrix metalloproteinase expression induced by collagen-coated and uncoated polypropylene meshes in a rat model. Am J Obstet Gynecol., 2011;205(1):82.e1-9. doi: 10.1016/j. ajog.2011.02.045. Epub $2011 \mathrm{Feb} 23$.

39. Dias F.G.F., Prudente A., Siniscalchi R.T., Campos de Vidal B., Zanettini Riccetto C.L. - Can highly purified collagen coating modulate polypropylene mesh immune-inflammatory and fibroblastic reactions? Immunohistochemical analysis in a rat model. Int Urogynecol J., October 2014.doi:10.1007/s00192-014-2529-0

40. Lukasiewicz A., Skopinska-Wisniewska J., Marszalek A., Molski S., Drewa T. - Collagen/Polypropylene composite mesh biocompatibility in abdominal wall reconstruction. Plast Reconstr Surg., 2013 May;131(5):731e-40e.doi:10.1097/PRS.0b013e3182865d2c.

41. van't Riet M., Burger J.W., Bonthuis F., Jeekel J., Bonjer H.J. - Prevention of adhesion formation to polypropylene mesh by collagen coating: a randomized controlled study in a rat model of ventral hernia repair. Surg Endosc., 2004;18(4):681-685. Epub 2004 Mar 19.

42. Cervigni M., Natale F., La Penna C., Saltari M., Padoa A., Agostini M. Collagen-coated polypropylene mesh in vaginal prolapse surgery: an observational study. Eur J Obstet Gynecol Reprod Biol., 2011;156(2) :223227.doi:10.1016/j.ejogrb.2011.01.027. Epub 2011 Mar 1.

43. Shulz D.D., Czeczko N.G., Malafaia O., Schulz G.J., Czeczko L.E., Garcia L.S., Dietz U.A. - Evaluation of healing prosthetic materials polyester mesh resorbable film an collagen elastin matrix/polypropylene used in rabbits abdominal wall defects. Acta Cir Bras., 2009;24(6):476-483.

44. Faulk D.M., Londono R., Wolf M.T., Ranallo C.A., Carruthers C.A., Wilemann J.D., Dearth C.L., Badylak S.F. - ECM hydrogel coating mitigates the chronic inflammatory response to polypropylene mesh. Biomaterials, 2014;35(30):8585-8595.doi:10.1016/j.biomaterials.2014.06.057. Epub 2014 Jul 16.

45. Dias F., Dos Santos A. - MP4-16 Can highly purified collagen coating modulate polypropylene mesh immune-inflammatory and fibroblastic 
reactions? Immunohistochemical analysis in rat model. The Journal of Urology, 2014;191(4) Suppl:e49.doi:10.1016/j.juro.2014.02.216.

46. Rudnicki M., Laurikainen E., Pogosean R., Kinne I., Jakobsson U., Teleman P. - Anterior colporrhaphy compared with collagen-coated transvaginal mesh for anterior vaginal wall prolapse: a randomised controlled trial. BJOG, 2014;121(1):102-110; discussion 110-111. doi:10.1111/1471-0528.12454. Epub 2013 Oct 1.

47. García-Pumarino R., Pascual G., Rodriguez M., Perez-Kohler B., Manuel Bellon J. - Do collagen meshes offer any benefits over preclude ${ }^{\circledR}$ ePTFE implants in contaminated surgical fields? A comparative in vitro and in vivo study. J Biomed Mater Res Part B, 2014;10(2):366-375.

48. Udpa N. - Effects Of Chitosan Coatings On Polypropylene Mesh For Pelvic Floor Reconstruction. Available at: http://hdl.handle. net/1813/36145

49. Gao Y., Krpata D.M., Criss C.N., Liu L., Posielski N., Rosen M.J., Novitsky Y.W. - Effects of mesenchymal stem cell and fibroblast coating on immunogenic potential of prosthetic meshes in vitro. Surgical Endoscopy, 2014;28(8):2357-2367.

50. Gao Y., Liu L., Blatnik J.A., Krpata D.M., Anderson J.M., Criss C.N., Posielski N., Novitsky Y.W. - Methodology of fibroblast and mesenchy- mal stem cell coating of surgical meshes: A pilot analysis. J Biomed Mater Res Part B, 2014;102B:797-805.

51. Lo T.S., Tan Y.L., Khanuengkitkong S., Dass A.K., Cortes E.F.M., Wu P.Y. - Assessment of Collagen-Coated Anterior Mesh Through Morphology and Clinical Outcomes in Pelvic Reconstructive Surgery for Pelvic Organ Prolapse. JMIG, 2014;21(5):753-761.

52. Rodriguez S. - Alloplastic materials and homografts in nasal reconstruction. April 2005. Available at: http://www.utmb.edu/otoref/grnds/ Nasal-Recon-050420/Nasal-Recon-050420.pdf

53. Winkler A.A., Soler Z.M., Leong P.L., Murphy A., Wang T.D., Cook T.A. - Complications Associated With Alloplastic Implants in Rhinoplasty. Arch Facial Plast Surg., 2012;14(6):437-441.doi:10.1001/archfacial.2012.583.

54. Berghaus A. - Implants for reconstructive surgery of the nose and ears. GMS Curr Top Otorhinolaryngol Head Neck Surg., 2007;6:Doc06.

55. Rai A., Datarkar A., Arora A., Adwani D.G. - Utility of High Density Porous Polyethylene Implants in Maxillofacial Surgery. Journal of Maxillofacial and Oral Surgery, 2014;13(1):42-46.

56. Giarenis I., Robinson D. - Prevention and management of pelvic organ prolapse. F1000Prime Rep., 2014;6:77. 